Bull. Austral. Math. Soc.

$47 \mathrm{~L} 30,47 \mathrm{~L} 35$

VOL. 64 (2001) [307-314]

\title{
DECOMPOSABILITY OF FINITE RANK OPERATORS IN CERTAIN SUBSPACES AND ALGEBRAS
}

\author{
JIANKUI LI
}

Let $\mathcal{S}$ be either a reflexive subspace or a bimodule of a reflexive algebra in $B(H)$, the set of bounded operators on a Hilbert space $H$. We find some conditions such that a finite rank $T \in \mathcal{S}$ has a rank one summand in $\mathcal{S}$ and $\mathcal{S}$ has strong decomposability. Let $\mathcal{S}(\mathcal{L})$ be the set of all operators on $H$ that annihilate all the operators of rank at most one in alg $\mathcal{L}$. We construct an atomic Boolean subspace lattice $\mathcal{L}$ on $H$ such that there is a finite rank operator $T$ in $\mathcal{S}(\mathcal{L})$ such that $T$ does not have a rank one summand in $\mathcal{S}(\mathcal{L})$. We obtain some lattice-theoretic conditions on a subspace lattice $\mathcal{L}$ which imply alg $\mathcal{L}$ is strongly decomposable.

\section{INTRODUCTION}

Let $H$ be a complex Hilbert space, $B(H)$ the set of bounded linear operators on $H$, and $F(H)$ the set of operators with finite rank. For convenience we disregard the distinction between a subspace of $H$ and the orthogonal projection onto it. Throughout, all subspaces will be assumed to be closed. By a subspace lattice on $H$, we mean a collection $\mathcal{L}$ of subspaces of $H$ with $(0), H$ in $\mathcal{L}$ and such that for every family $\left\{M_{r}\right\}$ of elements of $\mathcal{L}$, both $\cap M_{r}$ and $\vee M_{r}$ belong to $\mathcal{L}$, where $\vee M_{r}$ denotes the closed linear span of $\left\{M_{r}\right\}$. A totally ordered subspace lattice is called a nest. A complemented and distributive subspace lattice is called a Boolean lattice. An element $L$ of a subspace lattice $\mathcal{L}$ is called an atom if the condition $(0) \subseteq K \subseteq L(K \in \mathcal{L})$ implies either $K=(0)$ or $K=L$. A subspace lattice is atomic if each element of the lattice is the closed linear span of the atoms it contains.

For every subspace lattice $\mathcal{L}$ on $H$, we define $\operatorname{alg} \mathcal{L}$ by

$$
\operatorname{alg} \mathcal{L}=\{T \in B(H): T M \subseteq M, \text { for every } M \in \mathcal{L}\}
$$

Let $\mathcal{L}^{\perp}=\{I-P: P \in \mathcal{L}\}$. We have alg $\mathcal{L}^{\perp}=(\operatorname{alg} \mathcal{L})^{*}$. If $e, f$ are in $H$, then the rank one operator $x \mapsto f(x) e=(x, f) e$ is denoted by $e \otimes f$. If $K, L \in \mathcal{L}$, we denote by $L_{-}$the subspace $L_{-}=\vee\{M \in \mathcal{L}: L \nsubseteq M\}$, by $K_{\#}=\vee\left\{L \in \mathcal{L}: K \nsubseteq L_{-}\right\}$and by $K_{+}=\cap\{L \in \mathcal{L}: L \nsubseteq K\}$. By convention $H_{+}=\cap \emptyset=H,(0)_{-}=\vee \emptyset=(0)$. The

Received 13th February, 2001

We would like to thank Professors Don Hadwin and Rita Hibschweiler for their suggestions.

Copyright Clearance Centre, Inc. Serial-fee code: 0004-9727/01 $\$ A 2.00+0.00$. 
complete distributivity of $\mathcal{L}$ is equivalent to $K=K_{\#}$ for all $K \in \mathcal{L}$. An element $L$ in $\mathcal{L}$ is completely meet prime if $L \nsupseteq L_{+}$. An element $M$ in $\mathcal{L}$ is completely join prime if $M \nsubseteq M_{-}$.

If $M$ is a subset of $H$, we denote by $[M]$ the norm closure of $\operatorname{span}\{x: x \in M\}$. In this paper, " $\subseteq$ " is used for set inclusion while " $C$ " is reserved for proper inclusion. Let $R$ and $T$ be finite rank operators on $H$. We say that $R$ is a summand of $T$ if $\operatorname{rank} T=\operatorname{rank} R+\operatorname{rank}(T-R)$. Let $\mathcal{S}$ be a subset of $B(H)$. Then $\mathcal{S}$ is said to be decomposable if each finite rank operator in $\mathcal{S}$ is a sum of rank one operators in $\mathcal{S}$. We say that $\mathcal{S}$ is strongly decomposable if, for each $r>1$, each operator in $\mathcal{S}$ of $\operatorname{rank} r$ can be expressed as the sum of $r$ rank one operators in $\mathcal{S}$. A subspace $\mathcal{S}$ of $B(H)$ is said to be reflexive if whenever $T$ in $B(H)$ satisfies the condition $T x \in[\mathcal{S} x]$ for all $x \in H$, then $T$ is in $\mathcal{S}$.

Finite rank operators and rank one operators have been used extensively in the study of nest algebras and related non-self-adjoint reflexive algebras. By [5], we know that if $\mathcal{L}$ is a nest or an atomic Boolean subspace lattice on $H$, then alg $\mathcal{L}$ is strongly decomposable. These results were first proved in [16] and [12], respectively. These results were improved in [10]. Erdos and Power [3] proved that if $\mathcal{N}$ is a nest and $\mathcal{S}$ is a $\sigma$-weakly closed bimodule of alg $\mathcal{N}$, then $\mathcal{S}$ is strongly decomposable. In [6], Hopenwasser and Moore construct a totally atomic commutative subspace lattice $\mathcal{L}$ and a rank two operator in $\operatorname{alg} \mathcal{L}$ which cannot be written as a sum of rank one operators in $\operatorname{alg} \mathcal{L}$.

Let $\mathcal{S}$ be either a reflexive subspace or a bimodule of a reflexive algebra. For $T \in \mathcal{S} \cap F(H)$, we find some conditions such that $T$ has a rank one summand in $\mathcal{S}$. We also obtain some necessary and sufficient conditions which imply that $\mathcal{S}$ is strongly decomposable. We construct an atomic Boolean subspace lattice $\mathcal{L}$ on $H$ with three atoms for which there is a finite rank operator $T$ in $\mathcal{S}(\mathcal{L})$ such that $T$ does not have a rank one summand in $\mathcal{S}(\mathcal{L})$, where $\mathcal{S}(\mathcal{L})$ is the set of all operators on $H$ that annihilate all the operators of rank at most one in alg $\mathcal{L}$. This answers a question in [8] negatively. We obtain some lattice-theoretic conditions on a subspace lattice $\mathcal{L}$ which imply alg $\mathcal{L}$ is strongly decomposable. Theorems 2.12 and 2.13 generalise the main results of [10].

\section{MAIN RESULtS}

In [2], Erdos gives some necessary and sufficient conditions such that a reflexive subspace of $B(H)$ contains a rank one operator. In the following we obtain another equivalent condition.

LEMMA 2.1. Let $\mathcal{S}$ be a reflexive subspace of $B(H)$. Then $e \otimes f$ belongs to $\mathcal{S}$ if and only if $f \in(\operatorname{span}\{y: e \notin[\mathcal{S} y], y \in H\})^{\perp}$. 
Proof: Suppose that $e \otimes f \in \mathcal{S}$. For any $y$ in $H, e \otimes f(y)=(y, f) e \in[\mathcal{S} y]$. Hence if $e \notin[S y],(y, f)=0$. So $f \in(\operatorname{span}\{y: e \notin[S y], y \in H\})^{\perp}$.

Conversely, suppose $f \in(\operatorname{span}\{y: e \notin[\mathcal{S} y], y \in H\})^{\perp}$. Let $y \in H$. It follows that $e \otimes f(y)=(y, f) e \in[\mathcal{S} y]$. Since $\mathcal{S}$ is reflexive, it follows that $e \otimes f \in \mathcal{S}$.

The following Lemma will be used repeatedly.

LEMMA 2.2. ([5]) Let $T$ be a finite rank operator and let $A$ be a rank one operator in $B(H)$. Then $A$ is a summand of $T$ if and only if $A$ is of the form $(T y) \otimes$ $\left(T^{*} f\right.$ ) (or equivalently, $T(y \otimes f) T$ ), where $y$ and $f$ are vectors in $H$ and $(T y, f)=1$.

THEOREM 2.3. Suppose that $\mathcal{S}$ is a reflexive subspace of $B(H)$ and $T$ is a finite rank operator in $\mathcal{S}$. Then $T$ has a rank one summand in $\mathcal{S}$ if and only if there is a non-zero $e$ in $H$ such that $e \in T(H)$ and $e \notin \operatorname{span}\{T y: e \notin[\mathcal{S} y], y \in H\}$, where $T(H)$ is the range of $T$.

PRoof: Suppose that $0 \neq e \in T(H)$ and $e \notin \operatorname{span}\{T y: e \notin[\mathcal{S} y], y \in H\}$. Choose $g \in H$ such that $g \in(\operatorname{span}\{T y: e \notin[\mathcal{S} y], y \in H\})^{\perp},(e, g)=1$, and take $y \in H$ such that $T y=e$. For any $\tilde{y}$ satisfying $e \notin[S \tilde{y}]$, we have $\left(\tilde{y}, T^{*} g\right)=0$. It follows that $T^{*} g \in(\operatorname{span}\{\tilde{y}: e \notin[\mathcal{S} \tilde{y}], \tilde{y} \in H\})^{\perp}$. Using Lemma 2.1, $e \otimes T^{*} g=(T y) \otimes\left(T^{*} g\right) \in \mathcal{S}$. Using Lemma 2.2, $e \otimes\left(T^{*} g\right)=(T y) \otimes\left(T^{*} g\right)$ is a rank one summand of $T$ in $\mathcal{S}$.

Conversely, suppose that $T$ has a rank one summand in $\mathcal{S}$. By Lemma 2.2, there exist $m$ and $f$ in $H$ such that

$$
T(m \otimes f) T=(T m) \otimes\left(T^{*} f\right) \in \mathcal{S}
$$

and

$$
(T m, f)=1=\left(m, T^{*} f\right)
$$

Let $T m=e$. Using Lemma 2.1, we have $T^{*} f \in(\operatorname{span}\{y: e \notin[\mathcal{S} y], y \in H\})^{\perp}$. Hence for any $v \in \operatorname{span}\{y: e \notin[S y], y \in H\},\left(v, T^{*} f\right)=(T v, f)=0$. Since $(e, f)=(T m, f)=1$, it follows that $e \notin T(\operatorname{span}\{y: e \notin[\mathcal{S} y], y \in H\})=\operatorname{span}\{T y:$ $e \notin[\mathcal{S} y], y \in H\}$.

Corollary 2.4. Let $M$ and $N$ be nonzero subspaces of $H$ satisfying $M \cap N=$ (0) and $M \vee N=H$ and let $\mathcal{L}=\{(0), M, N, H\}$. Then every $\sigma$-weakly closed alg $\mathcal{L}$ bimodule $\mathcal{S}$ is strongly decomposable.

Proof: By [9, Theorem 2.2] and [1, Theorem 3.1], it follows that $\mathcal{S}$ is reflexive. By [4, Theorem 2], we know that $\mathcal{S}$ is determined by an order homomorphism $\phi$ of $\mathcal{S}$. Let $\phi$ be any order homomorphism of $\mathcal{L}$ and let

$$
\mathcal{M}=\{T \in B(H):(I-\phi(E)) T E=0, E \in \mathcal{L}\}
$$


By the symmetry of $M$ and $N$, we only need to consider that in the following cases $\mathcal{M}$ has strong decomposability.
(1) $\phi: M \mapsto M, N \mapsto(0)$,
(2) $\phi: M \mapsto N, N \mapsto(0)$,
(3) $\phi: M \mapsto N, N \mapsto M$,
(4) $\phi: M \mapsto M, N \mapsto N$,
(5) $\phi: M \mapsto H, N \mapsto(0)$.

For cases (1) to (4), we can easily prove the result using Theorem 2.3.

In case (5), $\mathcal{M}=\{T \in B(H): T N=0\}$. Let $P$ denote the projection on $N$. Then $T$ is in $\mathcal{M}$ if and only if $T P=0$. Hence $\mathcal{M}$ has strong decomposability.

If $\mathcal{L}$ is a subspace lattice on the Hilbert space $H$, let $\mathcal{S}(\mathcal{L})$ denote the set of all operators on $H$ that annihilate all the operators of rank at most one in alg $\mathcal{L}$, that is

$$
\mathcal{S}(\mathcal{L})=\{T \in B(H): \operatorname{tr}(T R)=0 \text {, for every } R \in \operatorname{alg} \mathcal{L} \text { of rank at most one }\} .
$$

Lemma 2.5. ([8]) For any subspace lattice $\mathcal{L}$ on $H$,

$$
\mathcal{S}(\mathcal{L})=\left\{T \in B(H): T(K) \subseteq K_{-} \text {for every } K \in \mathcal{L}\right\}
$$

LEMmA 2.6. ([8]) Let $\mathcal{L}$ be a subspace lattice on $H$ and $e, f \in H$. The following are equivalent.

(1) $e \otimes f \in \mathcal{S}(\mathcal{L})$,

(2) $e \in L$ and $f \in\left(L_{\#}\right)^{\perp}$ for some $L \in \mathcal{L}$.

THEOREM 2.7. Let $\mathcal{L}$ be a subspace lattice and let $T \in \mathcal{S}(\mathcal{L}) \cap F(H)$. Then $T$ has a rank one summand in $\mathcal{S}(\mathcal{L})$ if and only if there exists an $L \in \mathcal{L}$ such that $T(H) \cap L \nsubseteq T\left(L_{\#}\right)$.

Proof: Suppose that there exists $L \in \mathcal{L}$ such that $T(H) \cap L \nsubseteq T\left(L_{\#}\right)$. Let $e \in T(H) \cap L$ with $e \notin T\left(L_{\#}\right)$. Let $e=T y$. Choose $g \in\left(T\left(L_{\#}\right)\right)^{\perp}$ such that $(e, g)=(T y, g)=1$. We have, using Lemma 2.6

$$
(T y, g)=\left(y, T^{*} g\right)=1 \text { and } T(y \otimes g) T=(T y) \otimes\left(T^{*} g\right) \in \mathcal{S}(\mathcal{L}) .
$$

By Lemma 2.2, it follows that $T$ has a rank one summand in $\mathcal{S}(\mathcal{L})$.

Conversely, suppose $T$ has a rank one summand in $\mathcal{S}(\mathcal{L})$. By Lemma 2.2, there exist $e, f$ in $H$ such that

$$
T(e \otimes f) T=(T e) \otimes\left(T^{*} f\right) \in \mathcal{S}(\mathcal{L}) \text { and }(T e, f)=1 .
$$

By Lemma 2.6, there exists $L$ in $\mathcal{L}$ such that $T e \in L$ and $T^{*} f \in\left(L_{\#}\right)^{\perp}$. Since $T e \in L$, and $(T e, f)=1$ and for any $v \in L_{\#},(T v, f)=0$, we have that $T(H) \cap L \nsubseteq T\left(L_{\#}\right)$. 
Example 2.8. There is an atomic Boolean subspace lattice $\mathcal{L}$ with three atoms such that $\mathcal{S}(\mathcal{L})$ is not strongly decomposable.

Proof: Let $H$ be a finite-dimensional Hilbert space and let $A$ be an invertible operator in $B(H)$. Define $L_{1}=\{(x, 0,0): x \in H\}, L_{2}=\{(x, A x, 0): x \in H\}$ and $L_{3}=\{(x, A x, A x): x \in H\}$. By [1, Lemma 6.3], it follows that $\left\{L_{1}, L_{2}, L_{3}\right\}$ is the set of atoms of an atomic Boolean subspace lattice.

Define $T: L_{1} \rightarrow L_{2} \vee L_{3}$, by $(x, 0,0) \mapsto(0,0, P x), T: L_{2} \rightarrow L_{1} \vee L_{3}$, by $(x, A x, 0) \mapsto(0, P x, P x)$, and $T: L_{3} \rightarrow L_{2} \vee L_{1}$, by $(x, A x, A x) \mapsto(0, P x, 0)$, where $P$ is a nonzero finite rank projection in $B(H)$. We can extend $T$ to a bounded finite rank operator in $B(H \oplus H \oplus H)$. In fact $T(x, y, z)=\left(0, P A^{-1} y, P\left(x-A^{-1} z\right)\right)$, for every $x, y, z \in H$. By the definition of $T$, it follows that $T \in \mathcal{S}(\mathcal{L})$. We have that $T(H) \cap L_{1}=$ (0), $T(H) \cap L_{2}=(0)$ and $T(H) \cap L_{3}=(0)$. We can check that $T(H) \cap\left(L_{2} \vee L_{3}\right) \subseteq$ $T\left(L_{2} \vee L_{3}\right), T(H) \cap\left(L_{2} \vee L_{1}\right) \subseteq T\left(L_{2} \vee L_{1}\right)$ and $T(H) \cap\left(L_{1} \vee L_{3}\right) \subseteq T\left(L_{1} \vee L_{3}\right)$. Hence by Theorem 2.7, $T$ does not have a rank one summand in $\mathcal{S}(\mathcal{L})$, where $\mathcal{L}$ is the subspace lattice generated by $L_{1}, L_{2}$ and $L_{3}$.

REMARK. The above Example answers a question in $[8$, p.31] negatively.

Theorem 2.9. Suppose that $\mathcal{L}$ is a subspace lattice and $\operatorname{Rad}(\operatorname{alg} \mathcal{L})$ is the radical of $\operatorname{alg} \mathcal{L}$. Let $T \in \operatorname{Rad}(\operatorname{alg} \mathcal{L}) \cap F(H)$. Then $T$ has a rank one summand in $\operatorname{Rad}(\operatorname{alg} \mathcal{L})$ if and only if there exists an $M$ in $\mathcal{L}$ such that $T(H) \cap M \nsubseteq T\left(M_{-} \vee M\right)$.

Proof: Suppose that $T(H) \cap M \nsubseteq T\left(M_{-} \vee M\right)$. Choose $g$ in $\left(T\left(M_{-} \vee M\right)\right)^{\perp}$, $e$ in $H$ such that $(T e, g)=1$ and $T e \in M$. Then $\left(e, T^{*} g\right)=1,(T x, g)=\left(x, T^{*} g\right)=0$ for any $x \in M_{-} \vee M$. By $T^{*} g \in\left(M_{-} \vee M\right)^{\perp}, T e \in M$ and [7, Lemma 3], it follows that $(T e) \otimes\left(T^{*} g\right) \in \operatorname{Rad}(\operatorname{alg} \mathcal{L})$. By Lemma $2.2, T$ has a rank one summand in $\operatorname{Rad}(\operatorname{alg} \mathcal{L})$.

Conversely, suppose $T$ has rank one summand in $\operatorname{Rad}(\operatorname{alg} \mathcal{L})$. It follows that there exist $e, f \in H$ such that $T(e \otimes f) T=(T e) \otimes\left(T^{*} f\right) \in \operatorname{Rad}(\operatorname{alg} \mathcal{L})$. By [6, Lemma 3], there exists $M$ in $\mathcal{L}$ such that $T^{*} f \in\left(M_{-} \vee M\right)^{\perp},(T e, f)=1$. Hence $T(H) \cap M \nsubseteq T\left(M_{-} \vee M\right)$.

Let $\mathcal{L}_{1}$ and $\mathcal{L}_{2}$ be subspace lattices on Hilbert spaces $H_{1}$ and $H_{2}$, respectively. Then the ordinal sum $\mathcal{L}_{1}+\mathcal{L}_{2}$ is defined as the subspace lattice on $H_{1} \oplus H_{2}$ given by

$$
\mathcal{L}_{1}+\mathcal{L}_{2}=\left\{L \oplus(0): L \in \mathcal{L}_{1}\right\} \cup\left\{H_{1} \oplus M: M \in \mathcal{L}_{2}\right\}
$$

TheOREM 2.10. Let $\mathcal{L}_{1}$ and $\mathcal{L}_{2}$ be subspace lattices on Hilbert spaces $H_{1}$ and $H_{2}$. If $\mathcal{S}\left(\mathcal{L}_{1}\right)$ and $\mathcal{S}\left(\mathcal{L}_{2}\right)$ are strongly decomposable, then $S\left(\mathcal{L}_{1}+\mathcal{L}_{2}\right)$ is strongly decomposable.

Proof: Since

$$
\operatorname{alg}\left(\mathcal{L}_{1}+\mathcal{L}_{2}\right)=\left\{\left(\begin{array}{cc}
A_{1} & T \\
0 & A_{2}
\end{array}\right): A_{i} \in \operatorname{alg} \mathcal{L}_{i}, \text { for } i=1,2, T \in B\left(H_{2}, H_{1}\right)\right\},
$$


we have

$$
\mathcal{S}\left(\mathcal{L}_{1}+\mathcal{L}_{2}\right)=\left\{\left(\begin{array}{cc}
B_{1} & S \\
0 & B_{2}
\end{array}\right): B_{i} \in \mathcal{A}\left(\mathcal{L}_{i}\right), \text { for } i=1,2, \text { and } S \in B\left(H_{2}, H_{1}\right)\right\}
$$

Let $T$ be a finite rank operator in $S\left(\mathcal{L}_{1}+\mathcal{L}_{2}\right)$. Then

$$
T=\left(\begin{array}{cc}
T_{1} & S \\
0 & T_{2}
\end{array}\right), \text { where } T_{i} \in \mathcal{S}\left(\mathcal{L}_{i}\right) \text { for } i=1,2 \text { and } S \in B\left(H_{2}, H_{1}\right)
$$

Suppose $T_{1} \neq 0$. Since $\mathcal{S}\left(\mathcal{L}_{1}\right)$ is strongly decomposable, we may choose $e_{1} \in H_{1}$, $f_{1} \in H_{1}$ such that $T_{1}\left(e_{1} \otimes f_{1}\right) T_{1}$ is a rank one summand of $T_{1}$ in $\mathcal{S}\left(\mathcal{L}_{1}\right)$. Let $e=e_{1} \oplus 0$ and let $f=f_{1} \oplus 0$. For any $x=x_{1} \oplus x_{2} \in H_{1} \oplus H_{2},(x, f)=\left(x_{1}, f_{1}\right)$. It follows that

$$
T(e \otimes f) T=\left(\begin{array}{cc}
T_{1}\left(e_{1} \otimes f_{1}\right) T_{1} & T_{1}\left(e_{1} \otimes f_{1}\right) S \\
0 & 0
\end{array}\right) .
$$

By $(T e, f)=\left(T_{1} e_{1}, f_{1}\right)=1,(2.1)$ and $T(e \otimes f) T \in \mathcal{S}\left(\mathcal{L}_{1}+\mathcal{L}_{2}\right)$, it follows from Lemma 2.2 that $T(e \otimes f) T$ is a rank one summand of $T$ in $\mathcal{S}\left(\mathcal{L}_{1}+\mathcal{L}_{2}\right)$.

If $T_{1}=0$ and $T_{2} \neq 0$, we can similarly prove that $T$ has a rank one summand in $\mathcal{S}\left(\mathcal{L}_{1}+\mathcal{L}_{2}\right)$.

Suppose that $T_{1}=T_{2}=0$. Then $T=\left(\begin{array}{cc}0 & A \\ 0 & 0\end{array}\right)$. Since $B\left(H_{2}, H_{1}\right)$ is strongly decomposable, it follows that $T$ has a rank one summand in $\mathcal{S}\left(\mathcal{L}_{1}+\mathcal{L}_{2}\right)$.

Since $T$ is any finite rank operator in $\mathcal{S}\left(\mathcal{L}_{1}+\mathcal{L}_{2}\right)$, it follows that $\mathcal{S}\left(\mathcal{L}_{1}+\mathcal{L}_{2}\right)$ is strongly decomposable.

Let

$$
\mathcal{J}_{\mathcal{L}}=\left\{L \in \mathcal{L}: L \neq(0) \text { and } L_{-} \neq H\right\}, \mathcal{P}_{\mathcal{L}}=\left\{L \in \mathcal{L}: L \nsubseteq L_{-}\right\}
$$

By [13], we know that $L \in \mathcal{L}$ is completely meet prime if and only if $L=M_{-}$for some $M \in \mathcal{P}_{\mathcal{L}}$.

Lemma 2.11 . ([15, Lemma 2.3.1]) Let $K$ and $L$ be subspaces of $H$ and let $F=\sum_{i=1}^{n} e_{i} \otimes f_{i}$ be a rank $n$ operator in $B(H)$. If $F(L) \subseteq K$ and $f_{1} \notin L^{\perp}$, then $F$ can be written as $F=\tilde{e}_{1} \otimes f_{1}+\sum_{i=2}^{n} e_{i} \otimes \tilde{f}_{i}$ with $\tilde{e}_{1} \in K$.

THEOREM 2.12. Let $\mathcal{L}$ be a subspace lattice on $H$ such that $\mathcal{J}_{\mathcal{L}}=\mathcal{P}_{\mathcal{L}}$ and $\vee\left\{L: L \in \mathcal{J}_{\mathcal{L}}\right\}=H$. Then alg $\mathcal{L}$ is strongly decomposable.

Proof: Suppose that alg $\mathcal{L}$ is not strongly decomposable. Then there is an operator of rank $n>1 T=\sum_{i=1}^{n} e_{i} \otimes f_{i}$ in alg $\mathcal{L}$ such that $T$ does not have a rank one 
summand in $\operatorname{alg} \mathcal{L}$. By $H=\vee\left\{M: M \in \mathcal{J}_{\mathcal{L}}\right\}$, it follows that there exists an $M$ in $\mathcal{J}_{\mathcal{L}}$ such that $f_{1} \notin M^{\perp}$. By Lemma $2.11, T$ can be written as

$$
T=\tilde{e}_{1} \otimes f_{1}+\sum_{i=2}^{n} e_{i} \otimes \tilde{f}_{i},
$$

with $\tilde{e}_{1} \in M$. Let

$$
N=\cap\left\{L \in \mathcal{J}_{\mathcal{L}}: \widetilde{e}_{1} \in L\right\}
$$

Then $N \in \mathcal{J}_{\mathcal{L}}$ and $\tilde{e}_{1} \in N$.

Now we show that $\widetilde{e}_{1} \in N_{-}$. Suppose $\widetilde{e}_{1} \notin N_{-}$. Since $T^{*}=f_{1} \otimes \widetilde{e}_{1}+\sum_{i=2}^{n} \tilde{f}_{i} \otimes e_{i}$, by Lemma 2.11, we have that $T^{*}=g_{1} \otimes \widetilde{e}_{1}+\sum_{i=2}^{n} \widetilde{f}_{i} \otimes h_{i}$ with $g_{1} \in\left(N_{-}\right)^{\perp}$.

By $\tilde{e}_{1} \in N$ and $g_{1} \in\left(N_{-}\right)^{\perp}$, we have that $g_{1} \otimes \widetilde{e}_{1}$ is a rank one summand of $T^{*}$ in $\operatorname{alg} \mathcal{L}^{\perp}$. Hence $T$ has a rank one summand in alg $\mathcal{L}$. This is a contradiction.

Let $W=N_{-} \cap N$. We have $\tilde{e}_{1} \in W$ and $W \in \mathcal{J}_{\mathcal{L}}$. By the assumption, $W \subset N$ and $\widetilde{e}_{1} \in W$. This contradicts (2.2).

THEOREM 2.13. Let $\mathcal{L}$ be a subspace lattice on $H$ such that $\mathcal{J}_{\mathcal{L}}=\mathcal{P}_{\mathcal{L}}$ and $\cap\left\{L_{-}: L \in \mathcal{J}_{\mathcal{L}}\right\}=0$. Then alg $\mathcal{L}$ is strongly decomposable.

Proof: By [14, Proposition 2.1], it follows that

$$
\mathcal{J}_{\mathcal{L}^{\perp}}=\left\{\left(M_{-}\right)^{\perp}: M \in \mathcal{J}_{\mathcal{L}}\right\}
$$

Since $\mathcal{J}_{\mathcal{L}}=\mathcal{P}_{\mathcal{L}}$, for any $M \in \mathcal{J}_{\mathcal{L}}$, we have that $\left(M_{-}\right)^{\perp}$ is completely join prime. Hence for subspace lattice $\mathcal{L}^{\perp}$, we have $\mathcal{J}_{\mathcal{L}^{\perp}}=\mathcal{P}_{\mathcal{L}^{\perp}}$. By $\cap\left\{M_{-}: M \in \mathcal{J}_{\mathcal{L}}\right\}=(0)$, it follows that $\vee\left\{N: N \in \mathcal{J}_{\mathcal{L}^{\perp}}\right\}=H$. By Theorem 2.12, alg $\mathcal{L}^{\perp}$ is strongly indecomposable. It follows that $\operatorname{alg} \mathcal{L}$ is too.

Corollary 2.14. ([10]) Let $\mathcal{L}$ be a subspace lattice on $H$. If $\mathcal{L}$ satisfies one of the following conditions

(1) $\vee\left\{K: K \in \mathcal{J}_{\mathcal{L}}\right\}=H$ and for each $K$ in $\mathcal{J}_{\mathcal{L}}, K_{-} \vee K=H$,

(2) $\cap\left\{K_{-}: K \in \mathcal{J}_{\mathcal{L}}\right\}=(0)$ and for each $K$ in $\mathcal{J}_{\mathcal{L}}, K_{-} \vee K=H$, then $\operatorname{alg} \mathcal{L}$ is strongly decomposable.

If $\mathcal{L}$ is a completely distributive subspace lattice, by [11] we have $\vee\{L: L \in$ $\left.\mathcal{J}_{\mathcal{L}}\right\}=H$ and $\cap\left\{L_{-}: L \in \mathcal{J}_{\mathcal{L}}\right\}=(0)$. By Theorem 2.12 or Theorem 2.13 , we have the following result.

Corollary 2.15. ([15, Theorem 2.3.2]) Let $\mathcal{L}$ be a finite distributive subspace lattice on $H$ which satisfies $\mathcal{J}_{\mathcal{L}}=\mathcal{P}_{\mathcal{L}}$. Then alg $\mathcal{L}$ is strongly decomposable. 


\section{REFERENCES}

[1] S. Argyros, M.S. Lambrou and W.E. Longstaff, 'Atomic Boolean subspace lattices and applications to the theory of bases', Mem. Amer. Math. Soc. 445 (1991), 94.

[2] J.A. Erdos, 'Reflexivity for subspace maps and linear spaces of operators', Proc. London Math. Soc. 53 (1986), 582-600.

[3] J.A. Erdos and S.C. Power, 'Weakly closed ideals of nest algebras', J. Operator Theory 7 (1982), 219-235.

[4] D. Han, 'On $\mathcal{A}$-submodules for reflexive operator algebras', Proc. Amer. Math. Soc. 104 (1988), 1067-1070.

[5] K.J. Harrison and U.A. Mueller, 'Decomposability of reflexive cycle algebras', J. London Math. Soc. 51 (1995), 148-160.

[6] A. Hopenwasser and R. Moore, 'Finite rank operators in reflexive operators algebras', $J$. London Math. Soc. 27 (1983), 331-338.

[7] A. Katavolos and E. Katsoulis, 'Semisimplicity in operator algebras and subspaces', $J$. London Math. Soc. 42 (1990), 365-372.

[8] A. Katavolos, M.S. Lambrou and W.E. Longstaff, 'The decomposability of operators relative to two subspaces', Studia Math. 105 (1993), 25-36.

[9] J. Kraus and D. Larson, 'Reflexivity and distance formulae', Proc. London Math. Soc. 53 (1986), 340-356.

[10] J. Li, 'Decomposability of certain reflexive algebras', Houston J. Math. 23 (1997), 121-126.

[11] W.E. Longstaff, 'Strongly reflexive lattices', J. London Math. Soc. 11 (1975), 491-498.

[12] W.E. Longstaff, 'Operators of rank one in reflexive algebras', Canad. J. Math. 28 (1976), 19-23.

[13] W.E. Longstaff, J. Nation and O. Panaia, 'Abstract reflexive subspaces and completely distributive collapsibility', Bull. Austral. Math. Soc. 58 (1998), 245-260.

[14] W.E. Longtsaff and O. Panaia, ' $\mathcal{J}$-subspaces and subspace $M$-bases', Studia Math 139 (2000), 197-212.

[15] O. Panaia, Quasi-spatiality of isomorphisms for certain classes of operator algebras, Ph.D. Dissertation (University of Western Australia, 1995).

[16] J.R. Ringrose, 'On some algebras of operators', Proc. London Math. Soc. 15 (1965), 61-83.

Department of Mathematics

Hunan Normal University

Changsha, 410081

China
Department of Mathematics

University of New Hampshire

Durham, NH 03824

United States of America

e-mail: jkli@spicerack.sr.unh.edu 